\title{
Social media monitoring of mental health during disasters: \\ A scoping review of methods and applications
}

Samantha J. Teague ${ }^{1^{*}}$

Adrian B. R. Shatte ${ }^{2}$

Matthew Fuller-Tyszkiewicz ${ }^{1}$

Delyse M. Hutchinson 1,3,4,5

${ }^{1}$ Centre for Social and Early Emotional Development, School of Psychology, Deakin University, Geelong, Australia

${ }^{2}$ School of Engineering, Information Technology \& Physical Sciences, Federation University, Melbourne, Australia

${ }^{3}$ Murdoch Children's Research Institute, Centre for Adolescent Health, Royal Children's Hospital, Melbourne, Australia

${ }^{4}$ University of Melbourne, Department of Paediatrics, Royal Children's Hospital, Melbourne, Australia

${ }^{5}$ University of New South Wales, National Drug and Alcohol Research Centre, Sydney, Australia

*Corresponding author:

Dr Samantha Teague,

School of Psychology, Deakin University, 221 Burwood Hwy, Burwood, VIC Australia sam.teague@deakin.edu.au 


\section{Acknowledgements and Declarations}

\section{Financial Support}

This research received no specific grant from any funding agency, commercial or not-for-profit sectors.

\section{Conflict of Interest}

None.

\section{Contributions of Authors}

ST conceived of the study, participated in its design and coordination, performed the search and data extraction, interpreted the data, and drafted the manuscript; AS conceived of the study, participated in its design and coordination, contributed to the data extraction, contributed to the interpretation of the data, and helped to draft and revise the manuscript. MFT assisted with the interpretation of the data, and helped to draft and revise the manuscript; DH participated in the study's design and coordination, contributed to the interpretation of the data, and helped to draft and revise the manuscript. All authors read and approved the final manuscript. 


\begin{abstract}

\section{Background}

Difficulties in deploying mental health assessments during disasters have resulted in emerging research examining the use of social media as a population mental health monitoring tool. This review synthesises this literature, with particular focus on research methods and applications.

\section{Methods}

The field of social media monitoring of mental health during disasters was rapidly mapped using a scoping review methodology. Six interdisciplinary research databases were searched for relevant articles, with data extracted on the articles' applications and data collection and analysis methods. Articles were then synthesised via narrative review.
\end{abstract}

\title{
Results
}

Forty-seven papers were identified. Three application themes emerged, including: (i) estimating mental health burden; (ii) planning or evaluating interventions and policies, and (iii) knowledge discovery, where theories of human behaviour and mental health were evaluated. Applications across 30 mental health issues were identified, with mental health typically assessed using established linguistic dictionaries. Features extracted from social media data included linguistic, psycholinguistic, behavioural, and demographic features. Analytic techniques involved machine learning, statistical modelling, and qualitative analyses.

\section{Conclusions}

The application of social media monitoring has considerable potential for measuring the mental health impact on populations during disasters. As an emerging field, opportunities for further work were identified to improve mental health assessment methods, examine specific mental health conditions, and trial tools in real-world settings. Platforms integrated with such techniques may offer significant benefits for monitoring mental health in contexts where formal assessments are difficult to deploy, and may potentially be harnessed to monitor the impact of response efforts and intervention delivery.

Keywords: Social media, mental health, disaster, big data

Word count: 4,496 


\section{Introduction}

Disaster mental health has emerged as a critical public health issue, with increasing rates of both disasters and mental health impacts on the affected communities (Beaglehole et al., 2018; Leaning \& Guha-Sapir, 2013). Disasters are natural (e.g., earthquakes), technological (e.g., industrial accidents), or human-caused events (e.g., mass shootings) that have an acute and often unpredictable onset, are time delimited, and experienced collectively (McFarlane \& Norris, 2006). The unexpected and evolving nature of disasters makes it challenging to monitor the population's mental health in real-time. Capturing current, accurate, and representative information about a population's mental health during a disaster can assist in directing support to where it is most needed, monitor the impact of response efforts, and enable the delivery of targeted intervention. However, traditional methods of population-level mental health monitoring, such as large surveys of representative samples, can be logistically difficult to implement at short notice in an evolving and potentially deteriorating emergency context (Holmes et al., 2020).

Research has investigated the potential benefits of social media (also known as social networking sites, or SNS) data to capture the mental health status of impacted population groups during a disaster, and monitor their recovery over time (Gruebner et al., 2016; Jones et al., 2017). SNS data are advantageous due to: the low cost of implementation; ubiquity amongst the general population; the rich, real-time information that is shared by users (e.g., photos, text, video); and longitudinal assessment which permits modelling of time trends and the temporal sequencing of target variables (Hadi \& Fleshler, 2016). The large quantity and unstructured nature of SNS data also poses difficulties in terms of managing and extracting meaningful mental health information that is suitable for informing emergency response efforts.

A review capturing the strengths and weaknesses of the literature on disaster mental health monitoring via SNS is both pertinent and timely, given the availability of SNS analytic tools and the currently unfolding COVID-19 crisis (Aebi et al., 2021). Previous reviews have taken a more narrow focus by examining the health literature (e.g., Karmegam et al., 2019), yet substantial research has been published in other interdisciplinary areas (Shatte et al., 2019). Notably, research from computer science and engineering is particularly relevant, and may offer sophisticated methodological advances to address challenges specific to large, unstructured datasets obtained from SNS to indicate mental health outcomes (Chancellor \& De Choudhury, 2020; Shatte et al., 2019). This study aimed to conduct a scoping review of the interdisciplinary literature assessing mental health in disasters using SNS. Thus, this review aimed to: (i) identify how SNS data has been applied to monitor mental health during disasters, including the SNSs and mental health factors that have been investigated; and (ii) evaluate the methods used to extract 
meaningful or actionable findings, including the mental health assessment, data collection, feature extraction, and analytic techniques used.

\section{Method}

A scoping review methodology was selected to map the key concepts, main sources, and types of evidence available in the literature on mental health using SNS during disasters (Arksey \& O'Malley, 2005). The review was performed adhering to the Preferred Reporting Items for Systematic Reviews and Meta-Analyses extension for Scoping Reviews (PRISMA-ScR) guidelines (Tricco et al., 2018).

\section{Search strategy and study selection}

A broad search strategy was adapted from Shatte et al.'s (2019) review of machine learning applications in mental health. Both health and information technology research databases were selected, including Pubmed/MEDLINE, PsycINFO, Cochrane Library, Web of Science, IEEE Xplore, and the ACM Digital Library. Search terms were relevant to three themes: (i) mental health; (ii) SNS; and (iii) big data analytic techniques; with the search adapted to suit each database (see Supplementary File 1). The reference lists of all articles selected for review were manually searched for additional articles. The search was conducted on April 20th, 2021, with no time delimiters.

Article inclusion criteria were: (i) reported on a method or application of assessing mental health symptoms or disorders in a disaster, crisis, or emergency event; (ii) utilised SNS data, with SNS defined as any computer-mediated technology that facilitates social networks through user-generated content; (iii) published in a peer-reviewed publication; and, (iv) available in English. Articles were excluded if they: (i) did not report an original contribution to the research topic (e.g., commentaries, reviews); (ii) did not focus on a mental health application; (iii) did not have full text available (e.g. conference abstracts), and (iv) solely utilised other internet-based activities, such as web browser search behaviours. Articles were screened by the lead author, with the second author blindly double-screening $5 \%$ of title and abstract articles and $10 \%$ of full-text articles, obtaining a $100 \%$ agreement rate.

\section{Data extraction and analysis plan}

Data were extracted using a standardised template collating: (i) the aim and key findings of the research; (ii) the disaster event details; (iii) SNS, data collection methods and sample size; (iv) area of mental health focus and assessment methods; and (v) analytic methods used, including preprocessing steps, feature extraction, and algorithm details. To analyse the data, a narrative review synthesis method 
was selected to best capture the methods and applications in the identified studies. A meta-analysis was not considered appropriate for this review given the broad range of mental health issues and analytic techniques used in the studies identified.

--- Insert Figure 1 here ---

\section{Results}

\section{Overview of Article Characteristics}

The search strategy identified 4,075 articles, with 47 included in the review (see PRISMA-SC flowchart in Figure 1). The mean publication year was $2018(S D=2.44)$, with the earliest article published in 2013. Health crises were the most commonly researched disaster event ( $n=24$, including COVID-19, MERS, and SARS) followed by man-made disasters $(n=15$, including terrorist attacks, school or mass shootings, technological and transportation accidents, and armed conflict), and natural disasters ( $n=12$, including hurricanes, storms, floods, fires, earthquakes, tsunamis, and drought). Notably, the most commonly studied single disaster event was the COVID-19 pandemic $(n=22)$, with man-made disasters the most frequently studied disaster category prior to 2020. Disasters occurred most frequently in Asia $(n=17)$, followed by North America $(n=15)$, Europe $(n=6)$ and South America $(n=1)$. An additional nine studies used SNS data without any geographic restrictions. A total of 30 mental health issues were examined across the articles, with the most frequent being SNS users' affective response $(n=24)$, followed by anxiety $(n=8)$, depression $(n=7)$, stress $(n=3)$, and suicide $(n=3)$. The most common SNS was Twitter $(n=34)$, followed by Sina Weibo $(n=6)$, Facebook $(n=5)$, Youtube $(n=4)$, Reddit $(n=2)$, and other SNSs $(n=8)$. Overwhelmingly, the articles used an unobtrusive observational research design, with only two articles including any direct participation from users. Most articles reported the number of SNS posts $(n=44,93.6 \%)$; in contrast, few studies reported the unique number of users included in the analysis $(n=16,34 \%)$. The mean number of posts in the included studies was 1,644,760.58 $(S D=3,573,014.84$, Range $=17$ to $18,000,000)$, and the mean number of unique users was $164,318(S D=250,791.27$, Range $=49$ to 826,961$)$.

\section{Disaster Mental Health Applications}

Three disaster mental health application themes emerged: (i) estimating mental health burden $(n=33$, see Table 1), includes articles that identified posts from the affected disaster region to track or predict the changes to mental health over the disasters duration; (ii) planning or evaluating 
interventions/policies ( $n=9$, see Table 2), includes articles that monitored mental health via SNS as part of an intervention or policy evaluation; and finally (iii) knowledge discovery ( $n=5$, see Table 3 ), which includes a small number of articles that aimed to generate new insights into human behaviour using SNS in disaster contexts by developing theory and evaluating new hypotheses.

--- Insert Table 1 here ---

Estimating mental health burden. Articles that estimated the mental health burden after a disaster typically examined any negative affect present in posts using sentiment or affect dictionaries over the disaster's duration. For example, Gruebner et al., (2017) monitored the mental health of New Yorkers during the Hurricane Sandy disaster of 2012 using sentiment analysis of Twitter posts. Over the 11 days surrounding the hurricane's landfall, 24 spatial clusters emerged of basic emotions: before the disaster, clusters of anger, confusion, disgust and fear were present; a cluster of surprise was identified during the disaster; and finally a cluster of sadness emerged after the disaster. Expanding on this, Jones et al. (2016) examined the mental health trauma impact of school shooting events across three US college campuses using a quasi-experimental design, whereby an interrupted time series design was used with a control group and a reversal when the next shooting event occurred in the original control group's college. Increased negative emotion was observed after all three shooting events, particularly amongst users connected to the affected college campus within 2-weeks of the shooting. Finally, a few articles explored specific mental health conditions rather than general negative sentiment (e.g., depression in Sadasivuni \& Zhang, 2020; stress in Saha \& De Choudhury, 2017; and suicide in Woo et al., 2015). One notable study by Low et al. (2020) examined the impact of the initial stages of the COVID-19 pandemic on 15 mental health support groups on Reddit, allowing for disorder-specific monitoring and comparison. An increase in health anxiety and suicidality was detected across all mental health communities. Additionally, the $\mathrm{ADHD}$, eating disorder, and anxiety subreddits experienced the largest change in negative sentiment over the duration of the study, and became more homogenous to the health anxiety subreddit over time.

Planning or evaluating interventions/policies. Articles evaluating the mental health impact of disaster interventions or policies typically focused on the impact of public health measures during the COVID-19 crisis such as lockdowns, personal hygiene, and social distancing on SNS users' mental health. For example, Wang et al. (2020) compared the subjective wellbeing of Sina Weibo users in China experiencing lockdown versus those who were not, finding that lockdown policy had a positive impact on 
subjective wellbeing from the very low initial levels resulting from the pandemic. Next, two studies examined the impact of crisis communication on the mental health of SNS users impacted by a disaster, including government communication during the 2011 Great East Japan Earthquake/Fukushima Daiichi Nuclear Disaster and a school shooting event in the USA (Baek et al., 2013; Jones et al., 2017, respectively). Across the two studies, it was found that unclear or inconsistent official communication delivered via SNS led to a proliferation of rumours and public anxiety. Finally, three studies examined the mental health service needs of SNS users following disasters, including telehealth needs during the COVID-19 pandemic, mental illness stigma following a mass shooting, and tracking the support offered to disaster victims from a tornado and mass shooting (Budenz et al., 2018; K Glasgow et al., 2016, p. 20; Massaad \& Cherfan, 2020). Combined, these studies offer methods to assess the need for policies or interventions on specific mental health issues following a disaster by examining the access and availability of services to SNS users.

Knowledge discovery. The five articles that were classified as knowledge discovery aimed to evaluate theories of human behaviour and mental health during disasters using SNS data. This included examining the impact of psychological distance in the attention a disaster receives from SNS users (Van Lent et al., 2017), prosocial behaviour, coping, and desensitisation to trauma during the disaster (De Choudhury et al., 2014; Gaspar et al., 2016; Ye et al., 2020), and predicting recovery from SNS users purchasing behaviours and intentions (Shibuya \& Tanaka, 2018).

--- Insert Tables 2 \& 3 here ---

\section{Disaster Mental Health Methods}

Assessing mental health. Four methods were identified to assess mental health in SNS data. First, linguistic methods were the most frequently used $(n=26)$, such as the presence of keywords generated by the study authors (e.g., 'loneliness' and synonyms in Koh \& Liew, 2020), applying established dictionaries (e.g., the Linguistic Inquiry and Word Count (LIWC) dictionary in Lin et al., 2017)), or pre-trained language models (e.g., SKEP in Da \& Yang, 2020). Second, human assessment was used in 18 studies, with 11 using human annotators to conduct qualitative coding, typically for nuanced mental health information (e.g., type of social support received in Glasgow et al. 2016), and 7 studies interpreting a mental health topic from a topic modeling analysis. Next, two studies used mental health 
forum membership to indicate mental health problems (Low et al., 2020; Saha \& De Choudhury, 2017), with Saha and De Choudhury (2017) using the novel method of transfer learning from a classifier trained on a mental health subreddit ( $\mathrm{r} / \mathrm{stress}$ ) and a random sample of Reddit posts to identify posts with high stress on college-specific subreddits following gun-related violence on campus. Finally, mental health questionnaires were used in two studies; specifically, the PHQ-9 and GAD-7 in Zhang et al. (2020) and PWB Scale in Wang et al. (2020).

Very few studies implemented methods to improve data quality and validity of mental health assessments. Three studies included direct coding of posts using validated mental health measures. For example, Saha et al. (2017) directly annotated r/stress Reddit posts for high or low stress using the Perceived Stress Scale to develop a classifier. Gaspar et al. (2016) directly coded 885 tweets for coping during a food contamination crisis in Germany using Skinner et al.'s (2003) coping classification framework. Further, very few studies included a control or comparison group to delineate the relationship between the disaster and mental health. Comparison groups were identified in eight studies: five studies used a between-groups design typically selecting users from a different location to the disaster event as a comparison (Budenz et al., 2018; Da \& Yang, 2020; Kimberly Glasgow et al., 2014; Low et al., 2020; Wang et al., 2020), and three used a within-subjects comparison by comparing SNS users against their own data from a different point in time (Lin et al., 2017; Sadasivuni \& Zhang, 2020a; Saha \& De Choudhury, 2017).

Data collection and preprocessing. Data was primarily collected via the SNS's public streaming application programming interface (API) $(n=20)$. Digital archives and aggregation services of SNS data were the next most commonly used method $(n=10)$, such as the Harvard Center for Geographic Analysis Geo-tweet Archive (CGA) (Gruebner et al., 2016, 2018) and the TwiNL archive (van Lent et al., 2017). Finally, a few studies used other techniques including web crawlers $(n=8)$, third-party companies $(n=2)$, and other novel methods ( $n=2, n=6$ not reported) such as having participants download and share their usage patterns via Google Takeout (Zhang et al., 2020). In most studies ( $n=39)$, individual posts were the unit of analysis, rather than the user contributing those posts $(n=8)$. Sampling methods typically involved selecting posts or users with location data and key terms related to the crisis event. Identifying the location of users or posts included extracting location data from profile pages (e.g., Shibuya \& Tanaka, 2018), selecting users or posts with geotagged posts (e.g., Massaad \& Cherfan, 2020), or using users or posts with hashtags of the crisis location (e.g., De Choudhury et al., 2014). A few studies selected users that followed organisations local to the crisis event, including college campus subreddits or Twitter profiles (e.g., Jones et al., 2016; Saha \& De Choudhury, 2017). Key terms related to the crisis event 
included hashtags or keyterms of the event (e.g., \#Ebola in Van Lent et al., 2017) or mental health keywords (e.g., suicide or depression keywords in Woo et al., 2015).

Preprocessing steps to prepare the data for analysis were reported in $n=36$ articles, and typically involved translating posts into a single language (typically English) or removing posts in other languages, identifying and removing posts containing advertising or spam, and removing duplicate posts (e.g., retweets). Usernames, URLs and hashtags were either removed or normalised. Many studies also removed posts without keywords related to the crisis event, such as location or crisis-specific terms. Studies using NLP methods conducted additional preprocessing steps, including removing punctuation, stopwords, and non-printable characters, and stemming, lemmatizing, and tokenizing words. No studies reported how missing data were handled, or evidence of strategies to improve data quality, such as minimum thresholds of engagement with the SNS or mental health disclosure.

Feature extraction. Several features were extracted across studies, which have been grouped into linguistic, psycholinguistic, demographic, and behavioural features. Psycholinguistic features were the most frequently identified ( $n=34)$, and included sentiment (positive/negative/neutral), affect (positive/negative), time orientation (past/present/future tense), personal concerns (e.g., LIWC's work, money and death dictionaries), humour, valence, arousal, and dominance. These features were typically extracted using established lexicons, such as Stanford CoreNLP and LIWC. Some studies used direct coding of posts for psycholinguistic features, e.g., attitudes towards vaccinations (Du et al., 2018), and whether the post contained fear for self or others (van Lent et al., 2017). Linguistic features were present in 24 studies, and included n-grams, term frequency-inverse document frequency (tf-idf) statistics, bag of words, usernames, hashtags, URLs, and grammar and syntax features. Demographic features were less commonly used $(n=12)$, but typically involved the location associated with the SNS post. Some studies engineered location data into new metrics, e.g., negative emotion rate per local area surrounding the disaster in Karmegam and Mappillairaju (2020). A few studies identified the age and gender of users by extracting the information from user profiles (e.g., Li et al., 2020) or via age and gender lexicons (e.g., Genderize API, Lin et al., 2017).

While most studies used linguistic, psycholinguistic and demographic features only, a few studies also extracted additional features about users' behaviours. Behavioural features were identified in 7 studies, and included metrics such as a user's SNS post rate (e.g., Dore et al., 2017), sharing of news site URLs (Lin et al., 2017), and directly coded behaviours such as handwashing and social distancing (Song et al., 2017). Behavioural features also included the aspects of the user' social network (identified in $n=3$ studies), including the user's friend and follower counts (Song \& Xu, 2019; Massaad et al., 2020), and 
social interaction features (identified in $n=4$ studies) such as the use of @mentions (Lin et al., 2017; Song $\& \mathrm{Xu}, 2019)$ and retweets (Jones et al., 2017).

Analytic methods. A range of analytic methods were used across studies, including machine learning, statistical modelling, and qualitative techniques. The most common approach was trend analysis to examine temporal change in mental health before, during, and/or after the disaster event $(n=24)$. Geospatial analytic methods were also identified $(n=4)$, including geographic information system (GIS) methods to examine location-based differences in mental health factors during disasters. A few studies used machine learning classifiers to categorise posts or users into groups (e.g., high vs low stress in Saha and De Choudhury, 2017), namely support vector machine $(n=6)$, maximum entropy $(n=1)$, Multinomial Naive Bayes ( $n=2)$, Long Short Term Memory ( $n=2)$, Naive Bayes $(n=1)$, J48 $(n=1)$, Convolutional Neural Network $(n=1)$, tree ensemble $(n=1)$, and stochastic gradient descent $(n=1)$. Validation metrics for these studies involved k-folds cross-validation, with k's ranging from 5 to 10 . Finally, a number of studies implemented topic modelling $(n=12)$ and qualitative analytic approaches $(n=8)$ to identify themes in online discussions during the disaster. Topic modelling included latent Dirichlet allocation $(n=5), \mathrm{k}-$ means clustering $(n=3)$, hierarchical cluster analysis $(n=2)$, and other clustering methods $(n=3)$. Qualitative analyses included topic analysis $(n=1)$, thematic analysis $(n=2)$, touchpoint needs analysis $(n=1)$, content analysis $(n=1)$, and other coding techniques $(n=3)$.

Ethical considerations. There was limited discussion of the ethics of pervasive mental health monitoring in the identified studies $(n=18)$. Seven studies noted that their research protocol was reviewed and approved by an Institutional Review Board, and 5 stated that their research was exempt from ethical review. Some studies noted participant privacy concerns $(n=8)$, addressing this by anonymising usernames or handles, accessing only public information about users, and not publishing verbatim quotes from posts. Five articles provided information capable of reidentifying SNS users involved in their study, such as direct quotes from users and SNS post IDs. Two studies noted that their work complied with the SNS's data use policy. Only one study sought consent from SNS users to access their data.

\section{Discussion}

This study synthesised the literature assessing mental health in disasters using SNSs, highlighting current applications and methods. Research has predominantly focused on retrospectively monitoring the negative affect of SNS users local to a disaster area using established psycholinguistic dictionaries. Emerging research has assessed other public mental health issues, including the impact of news and government messaging, telehealth access, and mental health stigma. Analytic techniques are sophisticated 
in identifying relevant SNS users and modelling their changing mental health after the disaster. Overall, SNS offers a promising avenue to efficiently monitor public mental health in disasters, and is capable of overcoming many logistical challenges of traditional methods such as large sample sizes, before/during/after event data, and collection of comparison/control data.

As an emerging field, there are understandably significant gaps for future research to address. It is evident that mental health is broadly conceptualised by researchers as negative affect or distress, assessed using sentiment or affect dictionaries. However, it remains unclear whether spikes in posts with negative affect detected during and following disasters are clinically meaningful changes warranting intervention or the natural course of psychopathology following a distressing event (Galea \& Norris, 2006). More participatory research could address this issue by combining passive SNS monitoring with validated psychological measures capable of capturing both distress and dysfunction in the affected population (e.g., Zhang et al., 2020). Further, little research has examined the use of SNS to assess the impact of disasters on the mental health of vulnerable populations, including those with pre-existing mental health issues. Only one identified study compared how different mental health communities were impacted following a disaster (Low et al., 2020), finding both similarities and differences in responses between disorder-specific communities. Researchers may consider investigating how specific mental health conditions can be detected and monitored using SNS, particularly focusing on disorders that are likely to experience exacerbated symptomatology during disasters.

Beyond community mental health assessment, it is also clear that there is scope to improve the field by employing more robust research methods. The majority of studies used a pre-post crisis event design, but did not use a control or comparison group. SNS data offers many opportunities for comparing users, including users posting from an unaffected region and the user's own SNS data from different points in time. Such designs would assist in delineating the impact of the crisis event and response efforts on mental health assessments. Researchers should consider how to collect representative samples or control for demographic differences in analyses, for example, by matching sample data with census information, with very few studies considering the generalisability of results. Further, few studies considered the ethical implications of their research. Current ethical guidelines state that the large-scale and public nature of SNS data may enable such research to be exempt from review by ethics committees. Nevertheless, researchers need to be mindful of the sensitive nature of inferring mental health states using unvalidated methods from SNS data that may not be anonymous. The community needs to develop protocols for managing SNS users' privacy whilst maintaining high quality research practices (Ayers et al., 2018). Such practices could include removing ties between an account's posts and the user profile in publications, such as paraphrasing quotes and publishing meta-data rather than identifiable profiles or 
posts. Researchers also need to ensure that their study complies with the data use policies of the SNSs they are accessing, including user privacy requirements and the platform's preferred data access methods. To assist researchers in improving their study quality, Table 5 presents a reporting checklist of key methodological decisions to be considered. No included study met all of these reporting suggestions.

Finally, there are exciting avenues for future research to greatly progress the field. Emerging research has developed and evaluated new theories and hypotheses of mental health during disasters using SNS data, providing exciting advances in our understanding of how SNS data can be capitalised for knowledge discovery (e.g., De Choudhury et al., 2014; Ye et al., 2020). Such research should be encouraged by both computer and mental health scientists, given that other public health applications can be achieved by government organisations as part of their disaster response efforts (Galea \& Norris, 2006). No study was identified that evaluated the clinical utility of SNS mental health monitoring by developing and testing real-world disaster management tools. Translating tools into real-world settings will likely elicit new challenges that may not be present in the lab, particularly when applied across different clinical and emergency contexts. Another promising avenue for future research is to combine SNS mental health monitoring with intervention. This could be achieved by detecting individuals in need of support and directing them towards available interventions, including those designed for disaster contexts (e.g., psychological first aid/debriefing and crisis counselling), tailored mHealth and eHealth tools, or broader SNS-based interventions (North \& Pfefferbaum, 2013). Finally, the field would greatly benefit from more collaboration between mental health and computer science experts to bring nuance to the mental health conceptualisation and assessment alongside sophisticated analytic methods.

There are two key limitations to this review that should be considered alongside the study findings. First, the scoping review methodology entails a rapid and broad search to identify and map relevant literature. To balance these requirements, the search strategy used broad search terms and excluded non-English and non-peer reviewed literature (Pham et al., 2014). A more in-depth review would potentially capture additional relevant studies, but would be less feasible to complete and date quickly given the rapidly evolving nature of the field. Second, this study did not delineate how effectively SNS can be used to capture mental health impact during a disaster event as validation of mental health assessments against other measures were very limited. To address both of these limitations, future work could conduct an in-depth review of specific mental health issues, SNS, or disaster contexts, guided by the framework developed in the current study. 
In conclusion, there have been exciting advances in research aiming to monitor mental health during disasters using SNS. Overall, SNS data can be harnessed to infer mental health information useful for disaster contexts, including negative affect, anxiety, stress, suicide, grief, coping, mental illness stigma, and service access. Sophisticated analytic methods can be deployed to extract features from SNS data and model their geospatial and temporal distribution over the duration of the crisis event. As an emerging field, there are substantial opportunities for further work to improve mental health assessment methods, examine specific mental health conditions, and trial tools in real-world settings. Combined, such platforms may offer a useful avenue for monitoring mental health in contexts where formal assessments are difficult to deploy, and may potentially be harnessed for response effort monitoring and intervention delivery. 


\section{References}

Abd-Alrazaq, A., Alhuwail, D., Househ, M., Hai, M., \& Shah, Z. (2020). Top concerns of tweeters during the COVID-19 pandemic: A surveillance study. Journal of Medical Internet Research, 22(4). https://doi.org/10.2196/19016

Aebi, N. J., De Ridder, D., Ochoa, C., Petrovic, D., Fadda, M., Elayan, S., Sykora, M., Puhan, M., Naslund, J. A., Mooney, S. J., \& Gruebner, O. (2021). Can Big Data Be Used to Monitor the Mental Health Consequences of COVID-19? International Journal of Public Health, 66, 633451. https://doi.org/10.3389/ijph.2021.633451

Arksey, H., \& O’Malley, L. (2005). Scoping studies: Towards a methodological framework. International Journal of Social Research Methodology, 8(1), 19-32.

https://doi.org/10.1080/1364557032000119616

Ayers, J. W., Caputi, T. L., Nebeker, C., \& Dredze, M. (2018). Don’t quote me: Reverse identification of research participants in social media studies. Npj Digital Medicine, 1(1), 30. https://doi.org/10.1038/s41746-018-0036-2

Baek, S., Jeong, H., \& Kobayashi, K. (2013). Disaster Anxiety Measurement and Corpus-Based Content Analysis of Crisis Communication. 2013 IEEE International Conference on Systems, Man, and Cybernetics, 1789-1794. https://doi.org/10.1109/SMC.2013.309

Beaglehole, B., Mulder, R. T., Frampton, C. M., Boden, J. M., Newton-Howes, G., \& Bell, C. J. (2018). Psychological distress and psychiatric disorder after natural disasters: Systematic review and meta-analysis. The British Journal of Psychiatry, 213(6), 716-722. https://doi.org/10.1192/bjp.2018.210

Budenz, A., Purtle, J., Klassen, A., Yom-Tov, E., Yudell, M., \& Massey, P. (2018). The case of a mass shooting and violence-related mental illness stigma on twitter. Stigma and Health. https://doi.org/10.1037/sah0000155

Chancellor, S., \& De Choudhury, M. (2020). Methods in predictive techniques for mental health status on social media: A critical review. Npj Digital Medicine, 3(1). https://doi.org/10.1038/s41746-020- 
0233-7

Da, T., \& Yang, L. (2020). Local COVID-19 Severity and Social Media Responses: Evidence From China. IEEE Access, 8, 204684-204694. https://doi.org/10.1109/ACCESS.2020.3037248

De Choudhury, M., Monroy-Hernández, A., \& Mark, G. (2014). 'Narco’ emotions: Affect and desensitization in social media during the Mexican Drug War. Conference on Human Factors in Computing Systems - Proceedings, 3563-3572. https://doi.org/10.1145/2556288.2557197

Doré, B., Ort, L., Braverman, O., \& Ochsner, K. N. (2015). Sadness Shifts to Anxiety Over Time and Distance From the National Tragedy in Newtown, Connecticut. Psychological Science, 26(4), 363-373. https://doi.org/10.1177/0956797614562218

Galea, S., \& Norris, F. H. (2006). Public mental health surveillance and monitoring. In F. H. Norris, S. Galea, M. Friedman, \& P. Watson (Eds.), Methods for Disaster Mental Health Research (pp. 177-194). Guilford Press.

Gaspar, R., Pedro, C., Panagiotopoulos, P., \& Seibt, B. (2016). Beyond positive or negative: Qualitative sentiment analysis of social media reactions to unexpected stressful events. Computers in Human Behavior, 56, 179-191. https://doi.org/10.1016/j.chb.2015.11.040

Glasgow, K, Fink, C., Vitak, J., \& Tausczik, Y. (2016). 'Our Hearts Go Out': Social Support and Gratitude after Disaster. 2016 IEEE 2nd International Conference on Collaboration and Internet Computing (CIC), 463-469. https://doi.org/10.1109/CIC.2016.069

Glasgow, Kimberly, Fink, C., \& Boyd-Graber, J. (2014). ‘Our Grief is Unspeakable’: Automatically measuring the community impact of a tragedy. Proceedings of the 8th International Conference on Weblogs and Social Media, ICWSM 2014, 161-169.

Gruebner, O., Lowe, S. R., Sykora, M., Shankardass, K., Subramanian, S. V., \& Galea, S. (2017). A novel surveillance approach for disaster mental health. PLoS ONE, 12(7), 1-15. https://doi.org/10.1371/journal.pone.0181233

Gruebner, O., Lowe, S. R., Sykora, M., Shankardass, K., Subramanian, S. V., \& Galea, S. (2018). Spatiotemporal distribution of negative emotions in New York city after a natural disaster as seen in 
social media. International Journal of Environmental Research and Public Health, 15(10). https://doi.org/10.3390/ijerph15102275

Gruebner, O., Sykora, M., Lowe, S. R., Shankardass, K., Trinquart, L., Jackson, T., Subramanian, S. V., \& Galea, S. (2016). Mental health surveillance after the terrorist attacks in Paris. The Lancet, 387(10034), 2195-2196. https://doi.org/10.1016/S0140-6736(16)30602-X

Gupta, R., \& Agrawal, R. (2021). Are the concerns destroying mental health of college students?: A qualitative analysis portraying experiences amidst COVID-19 ambiguities. Analyses of Social Issues and Public Policy, asap.12232. https://doi.org/10.1111/asap.12232

Hadi, T. A., \& Fleshler, K. (2016). Integrating Social Media Monitoring Into Public Health Emergency Response Operations. Disaster Medicine and Public Health Preparedness, 10(5), 775-780. https://doi.org/10.1017/dmp.2016.39

He, Q., Du, F., \& Simonse, L. W. L. (2021). A Patient Journey Map to Improve the Home Isolation Experience of Persons With Mild COVID-19: Design Research for Service Touchpoints of Artificial Intelligence in eHealth. JMIR Medical Informatics, 9(4), e23238. https://doi.org/10.2196/23238

Holmes, E. A., O’Connor, R. C., Perry, V. H., Tracey, I., Wessely, S., Arseneault, L., Ballard, C., Christensen, H., Cohen Silver, R., Everall, I., Ford, T., John, A., Kabir, T., King, K., Madan, I., Michie, S., Przybylski, A. K., Shafran, R., Sweeney, A., ... Bullmore, E. (2020). Multidisciplinary research priorities for the COVID-19 pandemic: A call for action for mental health science. The Lancet Psychiatry, 7(6), 547-560. https://doi.org/10.1016/S22150366(20)30168-1

Hung, M., Lauren, E., Hon, E. S., Birmingham, W. C., Xu, J., Su, S., Hon, S. D., Park, J., Dang, P., \& Lipsky, M. S. (2020). Social Network Analysis of COVID-19 Sentiments: Application of Artificial Intelligence. Journal of Medical Internet Research, 22(8), e22590. https://doi.org/10.2196/22590

Jones, N. M., Brymer, M., \& Silver, R. C. (2019). Using big data to study the impact of mass violence: 
Opportunities for the traumatic stress field. Journal of Traumatic Stress, 32(5), 653-663. https://doi.org/10.1002/jts.22434

Jones, N. M., Thompson, R. R., Schetter, C. D., Silver, R. C., Dunkel Schetter, C., \& Silver, R. C. (2017). Distress and rumor exposure on social media during a campus lockdown. Proceedings of the National Academy of Sciences of the United States of America, 114(44), 11663-11668. https://doi.org/10.1073/pnas.1708518114

Jones, N. M., Wojcik, S. P., Sweeting, J., \& Silver, R. C. (2016). Tweeting negative emotion: An investigation of twitter data in the aftermath of violence on college campuses. Psychological Methods, 21(4), 526-541. https://doi.org/10.1037/met0000099

Karmegam, D., \& Mappillairaju, B. (2020). Spatio-temporal distribution of negative emotions on Twitter during floods in Chennai, India, in 2015: A post hoc analysis. International Journal of Health Geographics, 19(1). https://doi.org/10.1186/s12942-020-00214-4

Karmegam, D., Ramamoorthy, T., \& Mappillairajan, B. (2019). A Systematic Review of Techniques Employed for Determining Mental Health Using Social Media in Psychological Surveillance during Disasters. Disaster Medicine and Public Health Preparedness. https://doi.org/10.1017/dmp.2019.40

Khalid, H. M., Helander, M. G., \& Hood, N. A. (2013). Visualizing disaster attitudes resulting from terrorist activities. Applied Ergonomics, 44(5), 671-679. https://doi.org/10.1016/j.apergo.2012.06.005

Koh, J. X., \& Liew, T. M. (2020). How loneliness is talked about in social media during COVID-19 pandemic: Text mining of 4,492 Twitter feeds. Journal of Psychiatric Research, S0022395620310748. https://doi.org/10.1016/j.jpsychires.2020.11.015

Kumar, D. A., \& Chinnalagu, A. (2020). Sentiment and Emotion in Social Media COVID-19 Conversations: SAB-LSTM Approach. 2020 9th International Conference System Modeling and Advancement in Research Trends (SMART), 463-467.

https://doi.org/10.1109/SMART50582.2020.9337098 
Leaning, J., \& Guha-Sapir, D. (2013). Natural Disasters, Armed Conflict, and Public Health. New England Journal of Medicine, 369(19), 1836-1842. https://doi.org/10.1056/NEJMra1109877

Lee, H., Noh, E. B., Choi, S. H., Zhao, B., \& Nam, E. W. (2020). Determining Public Opinion of the COVID-19 Pandemic in South Korea and Japan: Social Network Mining on Twitter. Healthcare Informatics Research, 26(4), 335-343. https://doi.org/10.4258/hir.2020.26.4.335

Li, S., Wang, Y., Xue, J., Zhao, N., \& Zhu, T. (2020). The Impact of COVID-19 Epidemic Declaration on Psychological Consequences: A Study on Active Weibo Users. International Journal of Environmental Research and Public Health, 17(6). https://doi.org/10.3390/ijerph17062032

Li, X., Wang, Z., Gao, C., \& Shi, L. (2017). Reasoning human emotional responses from large-scale social and public media. Applied Mathematics and Computation, 310, 182-193. https://doi.org/10.1016/j.amc.2017.03.031

Lin, Y. R., Margolin, D., \& Wen, X. (2017). Tracking and analyzing individual distress following terrorist attacks using social media streams. Risk Analysis, 37(8), 1580-1605. https://doi.org/10.1111/risa.12829

Low, D. M., Rumker, L., Talkar, T., Torous, J., Cecchi, G., \& Ghosh, S. S. (2020). Natural Language Processing Reveals Vulnerable Mental Health Support Groups and Heightened Health Anxiety on Reddit During COVID-19: Observational Study. Journal of Medical Internet Research, 22(10), e22635. https://doi.org/10.2196/22635

Massaad, E., \& Cherfan, P. (2020). Social Media Data Analytics on Telehealth During the COVID-19 Pandemic. Cureus. https://doi.org/10.7759/cureus.7838

Mathur, A., Kubde, P., \& Vaidya, S. (2020). Emotional Analysis using Twitter Data during Pandemic Situation: COVID-19. 2020 5th International Conference on Communication and Electronics Systems (ICCES), 845-848. https://doi.org/10.1109/ICCES48766.2020.9138079

McFarlane, A., \& Norris, F. H. (2006). Definitions and concepts in disaster research. In F. H. Norris, S. Galea, M. Friedman, \& P. Watson (Eds.), Methods for Disaster Mental Health Research (pp. 319). Guilford Press. 
North, C. S., \& Pfefferbaum, B. (2013). Mental Health Response to Community Disasters: A Systematic Review. JAMA, 310(5), 507. https://doi.org/10.1001/jama.2013.107799

Oyebode, O., Ndulue, C., Adib, A., Mulchandani, D., Suruliraj, B., Orji, F. A., Chambers, C. T., Meier, S., \& Orji, R. (2021). Health, Psychosocial, and Social Issues Emanating From the COVID-19 Pandemic Based on Social Media Comments: Text Mining and Thematic Analysis Approach. JMIR Medical Informatics, 9(4), e22734. https://doi.org/10.2196/22734

Pellert, M., Lasser, J., Metzler, H., \& Garcia, D. (2020). Dashboard of Sentiment in Austrian Social Media During COVID-19. Frontiers in Big Data, 3, 32. https://doi.org/10.3389/fdata.2020.00032

Pham, M. T., Rajić, A., Greig, J. D., Sargeant, J. M., Papadopoulos, A., \& McEwen, S. A. (2014). A scoping review of scoping reviews: Advancing the approach and enhancing the consistency. Research Synthesis Methods, 5(4), 371-385. https://doi.org/10.1002/jrsm.1123

Pran, Md. S. A., Bhuiyan, Md. R., Hossain, S. A., \& Abujar, S. (2020). Analysis Of Bangladeshi People's Emotion During Covid-19 In Social Media Using Deep Learning. 2020 11th International Conference on Computing, Communication and Networking Technologies (ICCCNT), 1-6. https://doi.org/10.1109/ICCCNT49239.2020.9225500

Sadasivuni, S. T., \& Zhang, Y. (2020a). Clustering Depressed and Anti-Depressed keywords Based on a Twitter Event of Srilanka Bomb Blasts using text mining methods. 2020 IEEE International Conference on Humanized Computing and Communication with Artificial Intelligence (HCCAI), 51-54. https://doi.org/10.1109/HCCAI49649.2020.00014

Sadasivuni, S. T., \& Zhang, Y. (2020b). Using Gradient Methods to Predict Twitter Users' Mental Health with Both COVID-19 Growth Patterns and Tweets. 2020 IEEE International Conference on Humanized Computing and Communication with Artificial Intelligence (HCCAI), 65-66. https://doi.org/10.1109/HCCAI49649.2020.00017

Saha, K., \& De Choudhury, M. (2017). Modeling Stress with Social Media Around Incidents of Gun Violence on College Campuses. Proc. ACM Hum.-Comput. Interact., 1(CSCW). https://doi.org/10.1145/3134727 
Shatte, A. B. R., Hutchinson, D. M., \& Teague, S. J. (2019). Machine learning in mental health: A scoping review of methods and applications. Psychological Medicine, 1-23.

Shekhar, H., \& Setty, S. (2015). Disaster analysis through tweets. 2015 International Conference on Advances in Computing, Communications and Informatics (ICACCI), 1719-1723. https://doi.org/10.1109/ICACCI.2015.7275861

Shibuya, Y., \& Tanaka, H. (2018). A Statistical Analysis Between Consumer Behavior and a Social Network Service: A Case Study of Used-Car Demand Following the Great East Japan Earthquake and Tsunami of 2011. The Review of Socionetwork Strategies, 12(2), 205-236. https://doi.org/10.1007/s12626-018-0025-6

Song, J., Song, T. M., Seo, D.-C. C., Jin, D.-L. L., \& Kim, J. S. (2017). Social Big Data Analysis of Information Spread and Perceived Infection Risk During the 2015 Middle East Respiratory Syndrome Outbreak in South Korea. Cyberpsychology, Behavior and Social Networking, 20(1), 22-29. https://doi.org/10.1089/cyber.2016.0126

Tricco, A. C., Lillie, E., Zarin, W., O’Brien, K. K., Colquhoun, H., Levac, D., Moher, D., Peters, M. D. J., Horsley, T., Weeks, L., Hempel, S., Akl, E. A., Chang, C., McGowan, J., Stewart, L., Hartling, L., Aldcroft, A., Wilson, M. G., Garritty, C., ... Straus, S. E. (2018). PRISMA Extension for Scoping Reviews (PRISMA-ScR): Checklist and Explanation. Annals of Internal Medicine, 169(7), 467-473. https://doi.org/10.7326/M18-0850

Van Lent, L. G. G., Sungur, H., Kunneman, F. A., Van De Velde, B., \& Das, E. (2017). Too far to care? Measuring public attention and fear for ebola using twitter. Journal of Medical Internet Research, 19(6). https://doi.org/10.2196/jmir.7219

Vo, B.-K. H., \& Collier, N. (2013). Twitter Emotion Analysis in Earthquake Situations. International Journal of Computational Linguistics and Applications, 4(1), 159-173.

Wang, Y., Wu, P., Liu, X., Li, S., Zhu, T., \& Zhao, N. (2020). Subjective Well-Being of Chinese Sina Weibo Users in Residential Lockdown During the COVID-19 Pandemic: Machine Learning Analysis. Journal of Medical Internet Research, 22(12), e24775. https://doi.org/10.2196/24775 
Woo, H., Cho, Y., Shim, E., Lee, K., \& Song, G. (2015). Public Trauma after the Sewol Ferry Disaster: The Role of Social Media in Understanding the Public Mood. International Journal of Environmental Research and Public Health, 12(9), 10974-10983. https://doi.org/10.3390/ijerph120910974

Xu, Q., Shen, Z., Shah, N., Cuomo, R., Cai, M., Brown, M., Li, J., \& Mackey, T. (2020). Characterizing Weibo Social Media Posts From Wuhan, China During the Early Stages of the COVID-19 Pandemic: Qualitative Content Analysis. JMIR Public Health and Surveillance, 6(4), e24125. https://doi.org/10.2196/24125

Xue, J., Chen, J., Hu, R., Chen, C., Zheng, C., Su, Y., \& Zhu, T. (2020). Twitter Discussions and Emotions About the COVID-19 Pandemic: Machine Learning Approach. Journal of Medical Internet Research, 22(11), e20550. https://doi.org/10.2196/20550

Ye, Y., Long, T., Liu, C., \& Xu, D. (2020). The Effect of Emotion on Prosocial Tendency: The Moderating Effect of Epidemic Severity Under the Outbreak of COVID-19. Frontiers in Psychology, 11, 588701. https://doi.org/10.3389/fpsyg.2020.588701

Zhang, B., Zaman, A., Silenzio, V., Kautz, H., \& Hoque, E. (2020). The Relationships of Deteriorating Depression and Anxiety With Longitudinal Behavioral Changes in Google and YouTube Use During COVID-19: Observational Study. JMIR Mental Health, 7(11), e24012. https://doi.org/10.2196/24012

Zhou, X., Song, Y., Jiang, H., Wang, Q., Qu, Z., Zhou, X., Jit, M., Hou, Z., \& Lin, L. (2021). Comparison of Public Responses to Containment Measures During the Initial Outbreak and Resurgence of COVID-19 in China: Infodemiology Study. Journal of Medical Internet Research, 23(4), e26518. https://doi.org/10.2196/26518 


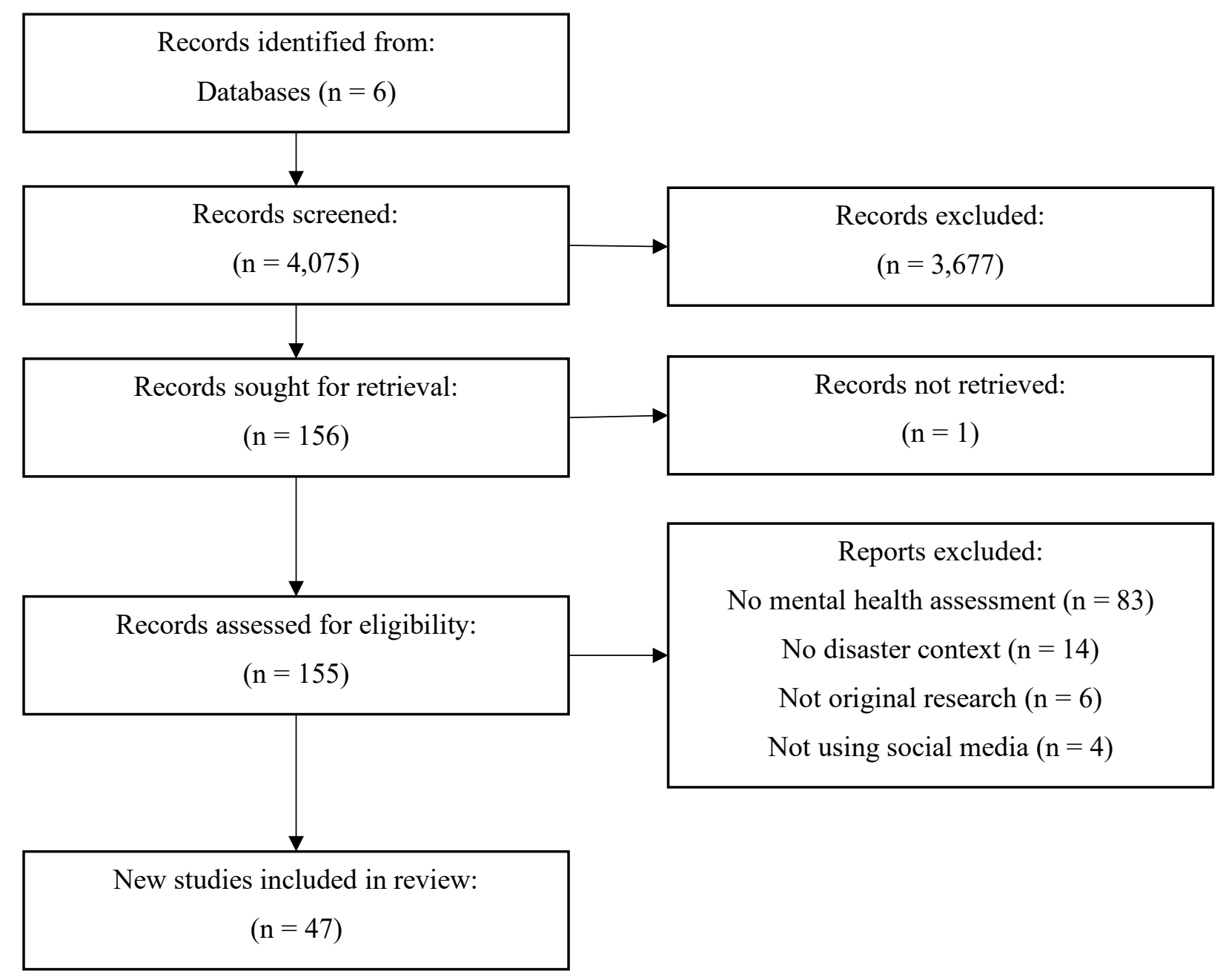

Figure 1: PRISMA-SC Flowchart 


\section{Table 1}

Summary of articles estimating mental health burden from social media during a disaster

\begin{tabular}{|c|c|c|c|c|c|c|}
\hline Article & Disaster Type & $\begin{array}{l}\text { Disaster } \\
\text { Year }\end{array}$ & Disaster Location & Mental Health Issue & $\begin{array}{c}\text { Social Media Platform } \\
\text { (SNS) }\end{array}$ & $\begin{array}{c}\text { Sample size } \\
\text { (Posts (Users)) }\end{array}$ \\
\hline \multicolumn{7}{|l|}{ Natural Disaster } \\
\hline (Gruebner et al., 2017) & Meteorological & 2012 & USA & Affective response & Twitter & 344,957 (NR) \\
\hline (Gruebner et al., 2018) & Meteorological & 2012 & USA & Affective response & Twitter & $1,018,140(\mathrm{NR})$ \\
\hline (Karmegam \& Mappillairaju, 2020) & Hydrological & 2015 & India & Affective response & Twitter & $5,696(\mathrm{NR})$ \\
\hline (Li et al., 2017) & $\begin{array}{l}\text { Geophysical, } \\
\text { Biological }\end{array}$ & $2009-2011$ & Japan, Haiti & Affective response & Twitter & $50,000(\mathrm{NR})$ \\
\hline (Shekhar \& Setty, 2015) & $\begin{array}{c}\text { Geophysical, } \\
\text { Climatological, } \\
\text { Hydrological }\end{array}$ & 2015 & Global & Affective response & Twitter & $60,519(\mathrm{NR})$ \\
\hline (Vo \& Collier, 2013) & Geophysical & 2011 & Japan & Affective response & Twitter & $70,725(\mathrm{NR})$ \\
\hline \multicolumn{7}{|l|}{ Man-Made Disaster } \\
\hline (Doré et al., 2015) & Active shooter & $2012-2013$ & USA & Affective response & Twitter & $43,548(\mathrm{NR})$ \\
\hline (Glasgow et al., 2014) & Active shooter & $2012-2013$ & USA & Grief & Twitter & $460,000(\mathrm{NR})$ \\
\hline (Gruebner et al., 2016) & Terrorist attack & 2015 & France & Affective response & Twitter & $22534(\mathrm{NR})$ \\
\hline (Jones et al., 2016) & Active shooter & 2014-2015 & USA & Affective response & Twitter & $325,736(6,314)$ \\
\hline (Jones et al., 2019) & Terrorist attack & 2015 & USA & Affective response & Twitter & $1,160,000(25,894)$ \\
\hline (Khalid et al., 2013) & Terrorist attack & NR & NR & Trauma & $\begin{array}{l}\text { Unspecified blogs and } \\
\text { discussion boards }\end{array}$ & $17(\mathrm{NR})$ \\
\hline (Lin et al., 2017) & Terrorist attack & 2015-2016 & France, Belgium & Affective response & Twitter & $18,000,000(\mathrm{NR})$ \\
\hline (Sadasivuni \& Zhang, 2020a) & Terrorist attack & 2019 & Sri Lanka & Depression & Twitter & $51,462(\mathrm{NR})$ \\
\hline (Saha \& De Choudhury, 2017) & Active shooter & $2012-2016$ & USA & Stress & Reddit & $113,337(\mathrm{NR})$ \\
\hline
\end{tabular}


(Woo et al., 2015)

\section{Epidemic/pandemic}

(Da \& Yang, 2020)

(Gupta \& Agrawal, 2021)

(Hung et al., 2020)

(Koh \& Liew, 2020)

(Kumar \& Chinnalagu, 2020)

(Lee et al., 2020)

(Li et al., 2020)

(Low et al., 2020)

(Mathur et al., 2020)

(Oyebode et al., 2021)

(Pellert et al., 2020)

(Pran et al., 2020)

(Sadasivuni \& Zhang, 2020b)
Accident

2011-2014

Korea

Suicide

Twitter

NR

$\begin{array}{lcc}\text { COVID-19 } & 2020 & \text { China } \\ \text { COVID-19 } & 2020 & \text { India } \\ \text { COVID-19 } & 2020 & \text { USA } \\ \text { COVID-19 } & 2020 & \text { Global } \\ \text { COVID-19 } & 2020 & \text { NR } \\ \text { COVID-19 } & 2020 & \text { Japan, Korea } \\ \text { COVID-19 } & 2020 & \text { China } \\ \text { COVID-19 } & 2018-2020 & \text { Global }\end{array}$

Affective response

Anxiety, depression, panic attacks, stress, suicide attempts

Psychological stress

$$
\text { Loneliness }
$$

Affective response

\section{Affective response}

Anxiety, depression,

$$
\text { indignation, happiness }
$$

Eating disorder, addiction, alcoholism, ADHD, anxiety, Autism, Bipolar Disorder, BPD, depression, health anxiety, loneliness, PTSD,

schizophrenia, social anxiety, suicide, broad mental health,

$$
\text { COVID-19 support }
$$

$\begin{array}{ccc}\text { COVID-19 } & 2019-2020 & \text { Global } \\ \text { COVID-19 } & 2020 & \text { Global }\end{array}$

Affective response

General mental health concerns

$\begin{array}{llc}\text { COVID-19 } & 2020 & \text { Austria } \\ \text { COVID-19 } & 2020 & \text { Bangladesh } \\ \text { COVID-19 } & 2020 & \text { Global }\end{array}$

\section{Sina Weibo}

340,456 (NR)

Twitter, Facbook,

WhatsApp, Blog

Twitter

NR

Twitter

Twitter, Facebook, YouTube, Blogs

$$
\text { Twitter }
$$

Sina Weibo

Reddit

$1,001,380(334,438)$

NR $(4,492)$

80,689 (NR)

4,951,289 (NR)

NR $(17,865)$

NR $(826,961)$

\section{Twitter}

30,000 (NR)

Twitter, YouTube,

Facebook, Archinect,

LiveScience, and

PushSquare

Twitter, Student chat $\quad 2,159,422(594,500)$ platform

Facebook

$1,120(\mathrm{NR})$

Twitter

$318,847(\mathrm{NR})$ 


\begin{tabular}{lcccccc} 
(Song et al., 2017) & MERS & 2015 & South Korea & Anxiety & $\begin{array}{c}\text { Twitter, } \\
\text { Blogs and discussion } \\
\text { boards }\end{array}$ \\
& & & & & A,671,695 (NR) \\
(Xu et al., 2020) & COVID-19 & $2019-2020$ & China & Affective response & Sina Weibo & $10,159(8,703)$ \\
(Xue et al., 2020) & COVID-19 & 2020 & Global & Affective response & Twitter & $4,196,020$ (NR) \\
(Zhang et al., 2020) & COVID-19 & 2020 & USA & Depression, anxiety & YouTube & 294,294 (49) \\
\hline
\end{tabular}

Notes: $\mathrm{NR}=$ Not reported; $\mathrm{ADHD}=$ attention-deficit/hyperactivity disorder; $\mathrm{BPD}=$ borderline personality disorder; $\mathrm{PTSD}=$ post-traumatic stress disorder; COVID-

$19=$ coronavirus disease 19 


\section{Table 2}

Summary of articles planning or evaluating interventions/policies from social media during a disaster

\begin{tabular}{|c|c|c|c|c|c|c|}
\hline & Disaster Type & $\begin{array}{c}\text { Disaster } \\
\text { Year }\end{array}$ & Disaster Location & Mental Health Issue & $\begin{array}{c}\text { Social Media } \\
\text { Platform (SNS) }\end{array}$ & $\begin{array}{c}\text { Sample size } \\
\text { (Posts (Users)) }\end{array}$ \\
\hline \multicolumn{7}{|l|}{ Natural } \\
\hline (Baek et al., 2013) & Geophysical, Accident & 2011 & Japan & Anxiety & Twitter & $179,431(\mathrm{NR})$ \\
\hline \multicolumn{7}{|l|}{ Man-Made } \\
\hline (Budenz et al., 2018) & Active shooter & 2017 & USA & Mental Illness Stigma & Twitter & $38,634(16,920)$ \\
\hline (K Glasgow et al., 2016) & Active shooter & 2011-2012 & USA & Coping and Social Support & Twitter & NR \\
\hline (Jones et al., 2017) & Active shooter & NR & USA & Psychological distress & Twitter & $7,824(2,515)$ \\
\hline \multicolumn{7}{|l|}{ Epidemic/pandemic } \\
\hline (Abd-Alrazaq et al., 2020) & COVID-19 & 2020 & Global & Affective response & Twitter & $167,073(160,829)$ \\
\hline (He et al., 2021) & COVID-19 & 2020 & Americas and Europe & Depression, mood instability & Youtube & 255 (NR) \\
\hline (Massaad \& Cherfan, 2020) & COVID-19 & 2020 & NR & Service access/needs & Twitter & 41,329 (NR) \\
\hline (Wang et al., 2020) & COVID-19 & 2020 & China & Subjective Well-Being & Sina Weibo & $\operatorname{NR}(5,370)$ \\
\hline (Zhou et al., 2021) & COVID-19 & 2020 & China & Affective response & Sina Weibo & 8,985,221 (NR) \\
\hline
\end{tabular}

Notes: $\mathrm{NR}=$ Not reported 


\section{Table 3}

Summary of articles discovering new knowledge and generating hypotheses from social media during disasters

\begin{tabular}{|c|c|c|c|c|c|c|}
\hline Article & Disaster Type & $\begin{array}{l}\text { Disaster } \\
\text { Year }\end{array}$ & $\begin{array}{l}\text { Disaster } \\
\text { Location }\end{array}$ & Mental Health Issue & $\begin{array}{c}\text { Social Media } \\
\text { Platform (SNS) }\end{array}$ & $\begin{array}{c}\text { Sample size } \\
\text { (Posts (Users)) }\end{array}$ \\
\hline \multicolumn{7}{|l|}{ Natural } \\
\hline (Gaspar et al., 2016) & Biological & 2011 & Germany & Coping & Twitter & 885 (NR) \\
\hline (Shibuya \& Tanaka, 2018) & Geophysical & 2011 & Japan & Anxiety & Facebook & $873,005(16,540)$ \\
\hline \multicolumn{7}{|l|}{ Man-Made } \\
\hline (De Choudhury et al., 2014) & War & $2010-2012$ & Mexico & $\begin{array}{c}\text { Anxiety, PTSD symptomatology, } \\
\text { affective response }\end{array}$ & Twitter & $3,119,037(219,968)$ \\
\hline \multicolumn{7}{|l|}{ Epidemic/pandemic } \\
\hline (Van Lent et al., 2017) & Ebola & 2014 & Netherlands & Affective response & Twitter & $4,500(\mathrm{NR})$ \\
\hline (Ye et al., 2020) & COVID-19 & 2020 & China & Prosociality, affective response & Sina Weibo & $569,846(387,730)$ \\
\hline
\end{tabular}


Table 5

Reporting checklist for social media analysis of mental health during disasters

\section{Research design and theoretical formulation \\ Type, place, and dates of disaster event \\ $\square \quad$ Use and selection of control or comparison group (e.g., matched comparison group, randomly sampled comparison group) \\ Theoretical justification for research question}

\section{Data collection}

$\square \quad$ Social media platform/s targeted

$\square \quad$ Data collection method specified

$\square \quad$ Sampling frame restrictions, e.g., dates, geolocation, keyword requirements

$\square \quad$ Number of unique social media posts and users

$\square \quad$ Management of sampling biases and confounding factors (e.g., matching demographics to census data)

\section{Mental health assessment}

$\square \quad$ Methods for assessing mental health status (e.g., sentiment analysis, LIWC affect dictionaries)

$\square \quad$ How ground truth was obtained (e.g., manual coding of social media posts, participant completion of validated psychological measures)

Clinical justification for assessment method

\section{Preprocessing and feature extraction}

Details of any manipulations to the data, e.g., text translation

Criteria for removed social media posts/users (e.g., spam/advertisements)

Use of minimum engagement thresholds

Handling of missing data

Transformation of data

Explicit number of features extracted and used in analysis

\section{Analysis}

Analytic technique/algorithm selection justification

$\square \quad$ Validation technique and metrics

$\square \quad$ Performance/fit of algorithm

$\square \quad$ Number of data points

$\square \quad$ Error analysis and explanation

Feature reduction techniques

\section{Ethical considerations}

Compliance with social media platform's data policy

$\square \quad$ Consideration of ethical research obligations, e.g., ethical review board approval

$\square \quad$ Minimising human exposure to participant data where possible (e.g., machine-based analyses)

$\square \quad$ Individual participant data not reported without consent (i.e., aggregate results reported only)

$\square \quad$ Use of public social media data only

$\square \quad$ Anonymisation of data to maintain participant privacy 


\section{Supplementary File 1: Search strategy}

Search details

Restrictions: English papers published in a peer-reviewed journal or conference proceeding.

Medical Subject Headings (MeSH)

1. Anxiety OR agoraphobia OR phobia* OR panic OR "posttraumatic stress" OR "mental health" OR "mental illness*” OR depress* OR "affective disorder*” OR bipolar OR "mood disorder*” OR psychosis OR psychotic OR schizophre* OR well-being OR wellbeing OR "quality of life" OR self-harm or "selfinjury" OR stress* OR distress* OR mood OR "body image" OR "eating disorder*”

2. “Social Media”, OR “Social Network” OR “Social Networking Sites” OR “web blog” OR newsgroup OR blog OR forum OR Twitter OR WhatsApp OR Facebook OR Weibo OR Snapchat OR YouTube OR Instagram OR MySpace OR Orkut OR WeChat OR Google+ OR Neverblog OR QQ OR Viber 3. "Machine Learning” OR "Data Mining” OR "Big Data” OR “Text Analysis” OR "Text Mining” OR "Predictive Analytics" OR "Deep Learning” OR "Natural Language Processing” OR “Artificial Intelligence" 